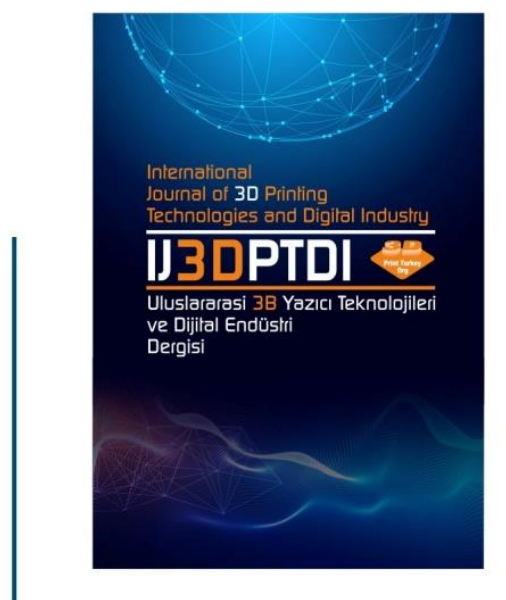

ULUSLARARASI 3B YAZICI TEKNOLOJILERI

VE DIJITAL ENDÜSTRI DERGISI

INTERNATIONAL JOURNAL QF 30 PRINTING TECHNOLOGIES AND DIGITAL INDUSTRY

IS5N:2602-3350 [Online]

URL: https://dergipark.org.tr/ij3dptdi

\title{
ADJUSTMENT OF ROD STRING VIBRATIONS IN DEEP- WELL PUMPS UPON IMPACT
}

Yazarlar (Authors): Javida Damirova *

Bu makaleye şu şekilde atıfta bulunabilirsiniz (To cite to this article): Damirova J., "Adjustment Of Rod String Vibrations In Deep-Well Pumps Upon Impact" Int. J. of 3D Printing Tech. Dig. Ind., 5(3): 353-360, (2021). 


\title{
ADJUSTMENT OF ROD STRING VIBRATIONS IN DEEP-WELL PUMPS UPON IMPACT
}

\author{
Javida Damirova ${ }^{a}$ iD * \\ ${ }^{a}$ Azerbaijan State Oil and Industry University, Information technologies and management, Control and system \\ engineering Department, AZERBAIJAN \\ * Corresponding Author: cavida.damirova@asoiu.edu.az
}

(Received: 29.06.2021; Revised: 03.08.2021; Accepted: 04.09.2021)

\begin{abstract}
In the article, studying the behavior of a rod strings is considered, the character of the change in dynamic pressure in rods is studied. On the basis of the equation fluctuation of a homogeneous core, the question on fluctuations of rod strings is studied more deeply. One of the most important problems in the operation of sucker rod pumps is the problem of selecting a sucker rod string. The growth of well depths requires not only a complete analysis of the operation of the existing equipment, but also the rational operation of such wells. Many researchers have dealt with the calculation of the dynamic forces acting on the sucker rods. Here, with the help of mathematical modeling, an analytical expression was obtained, which allows one to study the nature of changes in dynamic stresses in the rods (the equation 42), the problem of optimizing the choice of the dimensions and weight of the rods is formulated.
\end{abstract}

Keywords: Bars column, Dynamic load, Dynamic voltage, Fluctuations of a homogeneous core, Mathematical modeling, The weight of rod strings

\section{INTRODUCTION}

It should be noted that, in the US oilfield practice, the calculation of rod strings is based on the maximum working stress, not on the reduced stress, as in the CIS (Commonwealth of Independent State) countries or in the former USSR (Union of Soviet Socialist Republics). This leads to the characteristic shortcomings that are inherent in the method of calculating rod strings abroad. The essence of the calculation of the rod string is that for a given amount of liquid pumped out from a certain depth a day for a given dynamic level and properties of the liquid, it is required to select such a rod so that the working voltage in any of its sections does not exceed the maximum working for a given size and type of rods $[1,2]$. Consequently, it is necessary to investigate the behavior of the rod string upon impact, to study the nature of the change in the dynamic stresses in the rods. Therefore, it is advisable to study the issue of rod string vibrations upon impact in detail.

\section{MATERIALS AND METHODS}

The vibration equation of a homogeneous rod is written in the form [3-7]

$$
\frac{\partial^{2} u}{\partial t^{2}}=a^{2} \frac{\partial^{2} u}{\partial x^{2}} a x
$$

where $\mathrm{u}$ is the displacement of the section of a bar, i.e., in our case, a rod string, along its axis, and a function with coordinates $x$ and $t$ (time);

$a$ is the speed of propagation of a longitudinal wave in the rod, depending on the modulus of elasticity $E$ and the density $\rho$ of the rod material, $a^{2}=E / \rho$. 
The $x$-axis is assumed to be directed vertically downward. The origin of the axis is in the upper section of the rod string. The length of the rod in the unstretched state is $L$, the cross-sectional area is $F$. The upper end of the rod is assumed to be fixed, the lower end is free.

\section{EXPERIMENTAL PROCEDURES}

The problem will be solved in the following stages. Let the heavy rod strings, characterized by the above parameters $(E, F, L, \rho)$, are pre-stretched by the force $P_{0}$ applied to the lower section. At a moment in time $t=0$, the tensile force ceases to act and the rods begin to shrink. The lower section, having passed the path $L_{0}$ (the length of the plunger stroke), meets a rigid obstacle and stops.

It is necessary to identify and investigate the dynamic stresses arising from the sudden stop of the lower section. We solve the problem in two stages. First, consider the vibrations of a heavy rod, preliminarily separated from the state of equilibrium by the tensile force $P_{0}$. For this, it is necessary to solve equation (1) under certain boundary and initial conditions, which are set from the following considerations.

Since the upper end of the rod is fixed, during the whole process $u(0, t)=0$. At the moment of stretching by force $P_{0}$ in the intermediate section of the rod, which is spaced from the origin of coordinates by the value $x$, tensile stresses act

$\sigma=\frac{P_{0}}{F}+\frac{q(L-x)}{F}$

$q$ is the weight of a single rod length $(q=\gamma \cdot E)$. Bearing in mind that

$\sigma=E \cdot \frac{\partial u}{\partial x}$

We can write the following initial conditions

$\frac{\partial u}{\partial x}(x, 0)=\frac{P_{0}}{E F}+\frac{q(L-x)}{E F}$

or integrating from 0 to $x$.

$U(x, o)=\frac{P_{0} \cdot x}{E F}+\frac{q \cdot x(2 L-x)}{2 E F}$

Since at the initial moment the rods are motionless, then

$$
\frac{\partial u}{\partial t}(L, 0)=0
$$

Also, one more condition must be satisfied, expressing the fact of the disappearance of the tensile load at the lower end of the rod at $t>0$, this condition has the form

$$
\frac{\partial u}{\partial x}(L, t)=0
$$

Consider the solution of equation (1) under the following conditions:

$u(x, t)=0$ 


$$
\begin{aligned}
& u(x, o)=\frac{P_{0} \cdot x}{E F}+\frac{q \cdot x(2 L-x)}{2 E F}=f(x) \\
& \frac{\partial u}{\partial t}(L, 0)=0 \\
& \frac{\partial u}{\partial x}(L, t)=0
\end{aligned}
$$

The equation is solved by the Fourier method, i.e., we set the solution in the $u(x, t)=X(x) \cdot T(t)$ form. Substituting the product of $X$ and $\mathrm{T}$ into the original equation (1) and dividing both sides of the resulting equality by XT (assuming that $u \neq 0$ ), we obtain

$$
\frac{X^{\prime}}{X}=\frac{1}{a^{2}} \cdot \frac{T^{2}}{T}=-\lambda^{2}
$$

where $\lambda$ is an arbitrary constant.

Solving the resulting system of ordinary differential equations separately for $\mathrm{X}$ and for $\mathrm{T}$, we find that $u=\left(c_{1} \sin \lambda x+c_{2} \cos \lambda x\right) \cdot\left(c_{3} \sin \lambda a t+c_{4} \cos \lambda a t\right)$

Boundary condition (8) is satisfied only for $c_{2}=0$, and condition (10) - for $c_{3}=0$. Then the solution is somewhat simplified

$u=A \cos (\lambda a t) \cdot \sin (\lambda x)$

where $\mathrm{A}$ is a constant.

For condition (11) we find $\lambda$ :

$$
\frac{\partial u}{\partial x}(L, t)=A \lambda \cos (\lambda a t) \cdot \cos \lambda L=0
$$

Obviously, the latter can take place only for

$$
\lambda L=\frac{2 k+1}{2} \pi
$$

where $\kappa=0,1,2, \ldots$

As a result, the decision takes the form:

$$
u=\sum_{k=0}^{\infty} A_{k} \cos \left(\frac{2 k+1}{2 L} \pi a t\right) \sin \left(\frac{2 k+1}{2 L} \pi x\right)
$$

The coefficients $A_{\kappa}$ can be found by expanding the function $f(x)$ for condition (9) in a Fourier series in sines.

The decomposition coefficient is determined by the formula

$$
A_{k}=\frac{2}{L} \int_{0}^{L} f(x) \cdot \sin \left(\frac{2 k+1}{2 L} \pi x\right) d x=\frac{4 L^{2}}{\pi \lambda} \cdot \frac{(-1)^{k}}{(2 k+1)^{2}} ;
$$




$$
\int_{0}^{L} x^{2} \sin \left(\frac{2 k+1}{2 L} \pi x\right) d x=\frac{8 L^{2}(-1)^{k}}{\pi^{2}(2 k+1)^{2}}-\frac{16 L^{3}}{\pi^{2}(2 k+1)^{3}}
$$

and after simple calculations we obtain the final solution of equation (7)

$$
\begin{aligned}
& u=\frac{8 P_{0} L}{\pi^{2} E F} \sum_{k=0}^{\infty} \frac{(-1)^{k}}{(2 k+1)^{2}} \cos \left(\frac{2 k+1}{2 L} \pi a t\right) \sin \left(\frac{2 k+1}{2 L} \pi x\right)+ \\
& +\frac{16 q L^{2}}{\pi^{2} E F} \sum_{k=0}^{\infty} \frac{1}{(2 k+1)^{2}} \cos \left(\frac{2 k+1}{2 L} \pi a t\right) \sin \left(\frac{2 k+1}{2 L} \pi x\right)
\end{aligned}
$$

\section{RESULTS OF RESEARCH}

At the next stage of solving the problem, we will consider the dynamic stresses arising in the rod during a sudden stop of the lower section $(x=L)$. The study of the processes taking place in the lower part of the rod leads to quite interesting results.

We get $x=L$. Then

$$
\sin \frac{2 k+1}{2} \pi=(-1)^{k}
$$

Expression (19) takes the form

$$
u(L, t)=\frac{8 P_{0} L}{\pi E F} \sum_{(2 k+1)}^{\infty} \frac{1}{(2 k+1)^{2}} \cos \left(\frac{2 k+1}{2 L} \pi a t\right)+\frac{16 q L^{2}}{\pi^{2} E F} \sum_{k=0}^{\infty} \frac{(-1)^{k}}{(2 k+1)} \cos \left(\frac{2 k+1}{2 L} \pi a t\right)
$$

The sum of the obtained infinite series can be found by using the method of improving the convergence of Fourier series. The essence of the technique lies in the fact that an expansion of any simple function in a Fourier series is taken, and then, by successive integration of both the function itself and its expansion, new trigonometric series are obtained, the sum of which is easily found from the integration of the original function. Let us present the final results that are directly related to the problem under consideration.

$$
\begin{aligned}
& \sum_{k=0}^{\infty} \frac{\cos (2 k+1) \tau}{(2 k+1)^{2}}=\frac{\pi^{2}}{8}-\frac{\pi}{4} \tau \\
& \sum_{k=0}^{\infty} \frac{(-1)^{k} \cos (2 k+1) \cdot \tau}{(2 k+1)^{2}}=\frac{\pi^{3}}{32}-\frac{\pi}{8} \cdot \tau^{2}
\end{aligned}
$$

where $0 \leq \tau \leq \pi$.

Since the argument, $\tau=\frac{\pi}{2}, \frac{a t}{L}$ then (22) is valid only for the limits of variation of $t$ from 0 to $2 L / a$, i.e. within the travel time of the longitudinal wave from the lower end of the rod to the upper and back. Using (22), we obtain a solution to the problem in the following form:

$$
u(L, t)=\frac{P_{0}}{E F}(L-a t)+\frac{q}{2 E F}\left[L^{2}-(a t)^{2}\right]
$$

The moment of stopping of the lower section $t=\tau_{0}^{*}$ occurs when the displacement becomes equal to 
$u\left(L, \tau_{0}^{*}\right)=\frac{P_{0} L}{E F}+\frac{q L^{2}}{2 E F}-L_{k}$

where $L_{k}$ is the length of the free stroke of the plunger in the pump cylinder.

Substituting $\left(L, \tau_{0}^{*}\right)$ on the left side of (23) and solving the resulting quadratic equation for $a \tau_{0}^{*}$, we find

$a \tau_{0}^{*}=\frac{P_{0}}{q}\left(\sqrt{1+\frac{2 E F \cdot q}{P_{0}^{2}} L_{k}}-1\right)$

The speed of the plunger at the moment of impact is determined by differentiating (23) with respect to $\mathrm{t}$ and substituting instead of $\tau_{0}^{*}$ the value calculated from (25). As a result, we get:

$v_{\tau_{0}^{*}}=-a \cdot \frac{P_{0}}{E F} \cdot \sqrt{1+\frac{2 E F \cdot q}{F_{0}^{2}} \cdot L_{k}}$

where the "-" sign indicates that the movement is in the negative direction of the $x$-axis.

The shock load of the rod arising at the instantaneous stop of its lower section is found proceeding from the fact that the change in the kinetic energy of the system of material points is associated with the amount of motion by the following known relation

$$
\frac{d E_{k}}{d t}=K \frac{d v}{d t}
$$

where $E_{k}$ is the kinetic energy; $K$ is the amount of movement; $v$ - the speed of the bar in the considered section. The rod speed is determined by differentiation (19):

$$
u(x, t)=-\frac{8 q \cdot L \cdot a}{\pi E F} \sum_{k=0}^{\infty}\left[\frac{1}{(2 k+1)^{2}}-\frac{(-1)^{k} \pi P_{0}}{(2 k+1) 2 q L}\right] \cdot\left(\frac{2 k+1}{2 L} \pi a t\right) \sin \left(\frac{2 k+1}{2 L} \pi x\right)
$$

Kinetic energy of the rod

$$
E_{k}=\frac{q}{2 g} \int_{0}^{L} v^{2}(x, t) d x
$$

The operation of integrating the square of an infinite trigonometric series is facilitated by the fact that $\sin \left(\frac{2 k+1}{2 L} \pi x\right)$ is an orthogonal function in the interval $(0, L)$, i.e.

$\int_{0}^{L} \sin \left(\frac{2 m+1}{2 L} \pi x\right) \sin \left(\frac{2 n+1}{2 L} \pi x\right) d x= \begin{cases}0 & \text { at } m \neq n \\ L / 2 & \text { at } m=n\end{cases}$

or

$\int_{0}^{L} \sin ^{2}\left(\frac{2 k+1}{2 L} \pi x\right) d x=\frac{L}{2}$

where $m$ and $n$ are integers of the trigonometric series. 
Then the kinetic energy of the rod:

$$
E_{k}=\frac{8 q^{2} L^{2}}{\pi^{2} E F} \sum_{k=0}^{\infty}\left[\frac{1}{(2 k+1)^{2}}+\frac{(-1)^{k} \pi P_{0}}{q L(2 k+1)^{2}}+\frac{\left(\frac{\pi P_{0}}{2 q L}\right)^{2}}{(2 k+1)^{2}}\right] \cdot\left[1-\cos \left(\frac{2 k+1}{L} \cdot \pi a t\right)\right]
$$

Here it was meant $[8]$ that

$$
a^{2}=E \rho / \gamma, \quad a=\gamma \cdot F
$$

In view of the above, (32) takes the following form:

$$
\begin{aligned}
& E_{k}=\frac{P_{0}^{2}}{2 E F}(a t)\left[1+2 \frac{q L}{P_{0}}\left(\frac{a t}{L}\right)+\left(\frac{q L}{P_{0}}\right)^{2}\left(1-\frac{2}{3} \cdot \frac{a t}{L}\right)\right] \\
& \frac{d T}{d t}=\frac{P_{0}^{2} \cdot a}{2 E F}\left[1+2 \frac{q a \cdot t}{P_{0}}\left(2+\frac{q L}{P_{0}}\right)-2\left(\frac{q a t}{P_{0}}\right)^{2}\right]
\end{aligned}
$$

Now let's determine the amount of motion:

$$
K=\rho F \int_{0}^{L} v(x, t) d x
$$

Integrating (34) over $x$ and bearing in mind that $a^{2}=E / \rho$, we obtain:

$$
K=\frac{8 P_{0} L}{\pi^{2} a} \sum_{k=0}^{\infty} \frac{(-1)^{k} \sin \left(\frac{2 k+1}{2 L} \cdot \pi a t\right)}{(2 k+1)^{2}}+\frac{16 q L^{2}}{\pi^{2} a} \cdot \sum_{k=0}^{\infty} \frac{\sin \left(\frac{2 k+1}{2 L} \cdot \pi a t\right)}{(2 k+1)^{2}}
$$

Using the same technique with which the expansions (21) were found, we also find:

$$
\begin{aligned}
& \sum_{k=0}^{\infty} \frac{(-1)^{k}}{(2 k+1)} \cdot \sin \left(\frac{2 k+1}{2 L} \pi a t\right)=\frac{\pi^{2}}{8} \cdot \frac{a t}{L} \\
& \sum_{k=1}^{\infty} \frac{1}{(2 k+1)^{2}} \cdot \sin \left(\frac{2 k+1}{2 L} \pi a t\right)=\frac{\pi^{2}}{16} \cdot \frac{a t}{L}-\frac{\pi^{3}}{32}\left(\frac{a t}{L}\right)
\end{aligned}
$$

As a result, we get a very simple expression for $K$ :

$$
K=\left(P_{0}+q L\right) \cdot t-\frac{1}{2} q a t^{2}
$$

Substituting (37) and (34) into (27), we find the value of the acceleration $d v / d t$ :

$$
\frac{d v}{d t}=\frac{q}{2} \cdot \frac{1+2 \frac{q a t}{P_{0}}\left(2+\frac{q L}{P_{0}}\right)-2\left(\frac{q a t}{P_{0}}\right)}{q \cdot \frac{a t}{P_{0}}\left(1+\frac{q L}{P_{0}}-\frac{q a t}{P_{0}}\right)}
$$

Consequently, the dynamic load arising at the lower end of the rod string when the momentum changes from $K$ to 0 , 
$P_{\text {din }}=\frac{q L}{2} \cdot \frac{1+2 \frac{q a t}{P_{0}}\left(2+\frac{q L}{P_{0}}\right)-2\left(\frac{q a t}{P_{0}}\right)^{2}}{\frac{q a t}{P}\left(1+\frac{q L}{P_{0}}-\frac{q a t}{P_{0}}\right)}$

The time instant corresponding to the stop $\left(t=\tau_{0}^{*}\right)$ is defined (25):

$\frac{q a \tau_{0}^{*}}{P_{0}}=\sqrt{1+\frac{2 E F q L_{k}}{P_{0}^{2}}}-1$

Hence, we have:

$P_{\text {din }}=\frac{q L}{2} \cdot \frac{1+2\left(1+\frac{2 E F q L_{k}}{P_{0}^{2}}\right)^{1 / 2} \cdot\left(2+\frac{q L}{P_{0}}\right)-2\left[\left(1+\frac{2 E F q L_{k}}{P_{0}^{2}}\right)^{1 / 2}-1\right]^{2}}{\left[\left(1+\frac{2 E F q L_{k}}{P_{0}^{2}}\right)^{1 / 2}-1\right]\left(2+\frac{q L}{P_{0}}-\left(1+\frac{2 E F q L_{k}}{P_{0}^{2}}\right)^{1 / 2}\right]^{1 / 2}}$

Simplifying (41), we obtain

$$
P_{d i n} \approx q L\left(1+\frac{1}{2+\frac{q L}{P_{0}}-\sqrt{1+\frac{2 E F q L_{k}}{P_{0}^{2}}}}\right)
$$

\section{DISCUSSION OF EXPERIMENTAL RESULTS}

Calculations using the formula (42) showed that the dynamic loads arising in the rods can exceed the weight of the rod string by $30 \ldots 40 \%$. (Calculations by formula (42) were made in the author's dissertation work. This very voluminous work is therefore not indicated in the article) [9]. In this case, it is recommended to use the optimal technology for the production of rods from stronger steels and materials with high yield strengths. It is also permissible to choose rods with large diameters on top or hollow rods. It is also necessary to provide a lighter, more optimal operating mode that reduces load cycles and forces transmitted by the booms. Fig. 1 shows how the dynamic load changes depending on the initial tensile force $\mathrm{P}_{0}$,

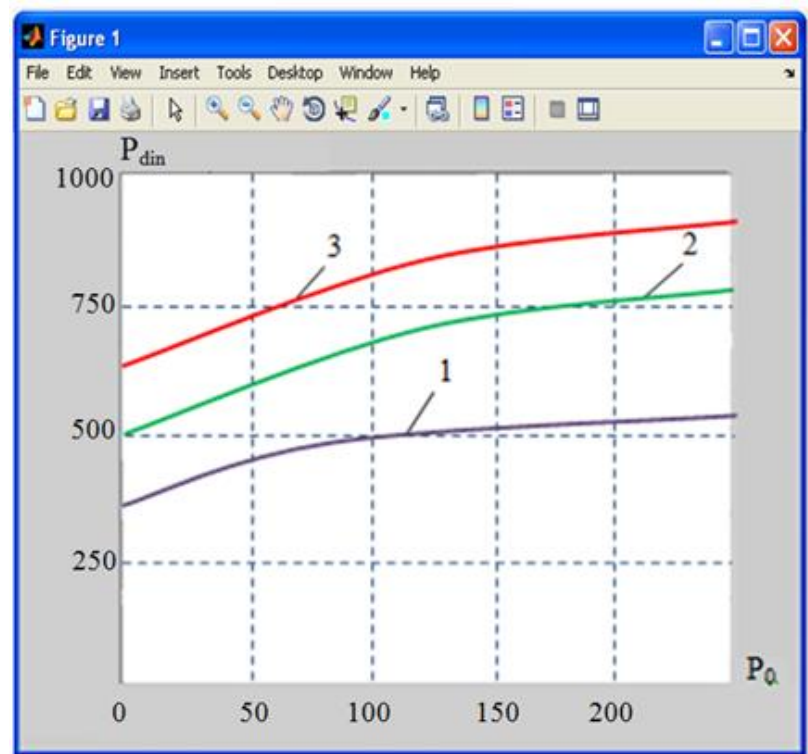

Figure 1. Change in dynamic load depending on the initial tensile force $P_{0}$, rod diameter $(\mathrm{d}, \mathrm{mm})$ $1-\mathrm{d}=22 \mathrm{~mm} ; 2-\mathrm{d}=28 \mathrm{~mm}, 3-\mathrm{d}=32 \mathrm{~mm}$. 


\section{CONCLUSIONS}

1. The question of vibrations of a rod string during impact was studied.

2. With the help of mathematical modeling, an analytical expression has been obtained, which allows one to study the nature of changes in dynamic stresses in the rods, the problem of optimizing the choice of the dimensions and weight of the rods is formulated.

3. Calculations using the formula (42) show that the dynamic loads arising in the rods can exceed the weight of the rod string by $30 \ldots 40 \%$.

4. A researcher who wants to work on this topic in the future should take into account and determine all the acting forces on the well rods, as well as their influence, in order to ensure the constant strength of the rods and their stabilization in a non-stationary mode.

\section{REFERENCES}

1. Muravyov I.M., Mishchenko I.T. Pumping operation of wells abroad. M.: Publishers Nedra, p. $238,1967$.

2. Adonin A.H. Extraction of oil by sucker rod pumps. M.: Publishers Nedra, p. 213, 1979.

3. Mammadov V.T., Damirova J.R. Investigation of stability losses of the packer with a girdle-cylindricalconical construction, International journal of current research, Vol. 10, Issue 2, pp.65391-65397, 2018.

4. Mammadov V.T., Aslanov C.N., Damirova J.R. Research of the unstable movement adjustment of sucker rod pump Engineering Studies, Taylor \&Francis, Vol.9 Issue 3, Pages 592-600, 2017.

5. Yusifov S.I., Mammadov V.T., Damirova J.R. Regulation of The Influence of The Dynamic Level on The Filling Factor of The Sucker Rod Pump, Scientific and Technical Journal, Oilfield business, Moscow: JSC VNIIOENG, Vol. 2, pp. 37-40, 2015.

6. Makushkin D.O. Calculation and design of machines and equipment for oil and gas fields [Electronic resource]: Textbook (in Russian) Siberian Federal University, p.156, 2009.

7. Kerimov Z.G. Drill string dynamic calculations. M.: Publishers Nedra, p.160. 1970.

8. Gradshtein I.S. and Ginger I.M. Tables of Integrals of Sums, Series and Products. Moscow: Fizmatizdat, p. 379, 1963.

9. Damirova J.R. Controlling the operating mode of a rod well pump unit according to a dynamic level. Dissertation for the degree of doctor philosophy on technology, Baku - 2018, p.173 